\title{
Climate Change and Future Analysis: Is STATIONARITY DYING?
}

\author{
Bruce A. McCarl, Xavier Villavicencio, and Ximing Wu
}

Economists often do risk analysis in support of management decisions. Commonly, such analyses are based on probability distributions arising from historical data where the distributions developed are based on at least a partial assumption of stationarity. For example, in water-based risk analysis one typically assumes the distribution is stationary, and uses the 100 year drought. In yield-related analyses analysts typically assume the mean is changing with time (proxying for technological progress along with monetary inflation) but that the variance is stationary.

Climate change may alter distribution stationarity (as asserted in a water setting by Milly et al. 2008). Evidence exists that climate change will shift the mean and variance of crop yields, challenging the stationarity assumption. If so, risk analysis would need to use distributions with nonstationary means and variances along with possibly shifting higher order moments. Here we examine stationarity and crop yields, statistically examining historical crop yield stationarity variability allowing both mean and variance to be affected. Finally, we evaluate stationarity under projected climate change scenarios.

\section{Background on Climate Change and Yields}

The influence of climate change on agricultural crop yields has been widely studied, as reviewed in documents such as the Intergovernmental Panel on Climate Change

Seniority of authorship is shared. The authors are Distinguished Professor and Regents Professor, research associate, and assistant professor, Department of Agricultural Economics, Texas A\&M University.

This article was presented in a principal paper session at the 2008 AAEA annual meeting in Orlando, FL. The articles in these sessions are not subjected to the journal's standard refereeing process. assessments $(2007,2001)$ or the U.S. National Assessment (Reilly et al. 2002). Many studies indicate that climate change alters mean yields (e.g., Adams et al. 1990; Reilly et al. 2002; Deschenes and Greenstone 2007) and/or land values (Mendelsohn, Nordhaus, and Shaw 1994). Chen, McCarl, and Schimmelpfennig (2004) also indicate that in addition to climate change affecting mean yields, it will contribute to a change in crop yield variability, while Mearns, Rosenzweig, and Goldberg (1992) provide crop simulation results to the same point.

\section{Model Specification}

The Chen, McCarl, and Schimmelpfennig (2004) study employs an estimation method based on the Just-Pope production function (1978) that allows statistical determination of the influence of climate on yield mean and variance and we will use that here but develop a richer specification. For temperature we use both its mean and variance during the growing season as exogenous variables. In addition we include average precipitation along with a precipitation intensity index and the Palmer Drought Severity Index (PDSI). Also, we incorporate interaction between regions and weather conditions. We pool data from 1960 through 2007 . We separate time invariant state-specific effects, time trends of the constructed panel. Also a linear and a quadratic trend were included to incorporate the effect of technological progress with the possibility of decreasing marginal returns.

The specification ala Just and Pope (1978) has the following form:

$$
y=f(X, \beta)+h(X, \alpha) \varepsilon
$$


where $y$ is crop yield; $f(\cdot)$ is an average production function; $X$ is a set of independent variables; and $\alpha$ and $\beta$ are unknown parameters to be estimated. In addition, $h(\cdot)$ is a function that accounts for explicit variable-dependent heteroskedasticity, allowing yield variability as a function of observed covariates. Under the assumption that the error term $\varepsilon$ is distributed with mean zero and unitary variance, $h^{2}(\cdot)$ is the yield variance.

To estimate the function we used feasible generalized least squares (FGLS). Following the basic Just-Pope procedure as follows: $(a)$ Estimate the model by ordinary least squares (OLS) and get the residuals; (b) Regress the logarithm of squared residuals against $X$ as independent variables; $(c)$ Get the predicted values of those residuals, which are calculated as the antilogarithm of the predictions from step $(b)$. They are consistent estimates of the variances; and $(d)$ Estimate the original model by weighted least squares (WLS), using the squared root of the variance predictions as weights.

\section{Data Set}

Our data set involved U.S. crop yields by state for corn, cotton, sorghum, soybeans, and winter wheat using observations from 1960 to 2007 from the National Agricultural Statistics Service (NASS). State-level climate data were obtained from the National Oceanic and Atmospheric Administration (NOAA) website. This included temperature means and standard deviations during the growing season. For rainfall we used total yearly precipitation. We also constructed a measure of precipitation intensity that was the ratio of total precipitation from the month with the highest amount of precipitation to the yearly total. This measure ranges from $1 / 12$ (uniformly intense) to 1 (one month gets all yearly rain).

In addition we included the PDSI, which indicates the severity of a wet or dry spell. This index generally ranges from -6 to +6 , with negative values denoting dry spells and positive values indicating wet spells.

\section{Estimation Results}

In this section we discuss in detail our estimation methods and results. We first test the hypothesis of panel unit roots, under which the classical inferences are generally invalid. This hypothesis is rejected. We then proceed to estimate the proposed model using the Fixed Effects model estimator for the stochastic production function.

\section{Panel Unit Roots}

Classical inference relies on the assumption of integration of order zero $I(0)$ of the involved series. Granger and Newbold (1974) showed that deterministic and stochastic trends in the series can induce spurious correlation between variables; as a result, we can obtain correlations between variables that are increasing for different reasons. The inclusion of time trends to control for this issue may not solve the problem when spurious correlation is present.

For these reasons it is necessary to test the presence of unit root for each variable prior to estimating the model explained above. If the series are found to be $I(1)$, they must be differenced before being included in the model. Traditional unit root tests are used to deal with testing one temporal series at a time; however, relatively new tests are available to test for unit roots of all cross-sections using the panel structure as a whole.

The objective is to test whether a given series is nonstationary for all the individual units (states in our case). We assume that the series follows a general panel data model structure (for $i$ states, and $t$ periods)

$$
\begin{aligned}
y_{i t} & =\left(1-\phi_{i}\right) \mu_{i}+\phi_{i} y_{i, t-1}+\varepsilon_{i t}, \\
i & =1, \ldots, N ; \quad t=1, \ldots T
\end{aligned}
$$

where $y_{i t}$ represents the variable to be tested, $\mu_{i}$ is a state-specific constant, $\phi_{i}$ is a statespecific parameter, and $\varepsilon_{i t}$ is an error term. This equation can be expressed as: $\Delta y_{i t}=\alpha_{i}+$ $\beta_{i} y_{i, t-1}+\varepsilon_{i t}$. We want to test if $\phi_{i}=1$ for all $i$, which is equivalent to the null hypothesis of panel unit root: $H_{0}: \beta_{i}=0$ for all $i$.

We performed two kinds of panel unit root tests. Im, Pesaran, and Shin (2003) proposed one in which the alternative hypothesis is that the series is stationary for some individuals and not stationary for others: $H_{1}: \beta_{i}<0, i=$ $1, \ldots, N_{1}, \quad \beta_{i}=0, i=N_{1}+1, \ldots, N$. In addition Levin, Lin, and Chu (2002) proposed a test in which the alternative hypothesis is that the series is stationary for all the individuals, say $H_{1}: \beta_{i}<0, i=1, \ldots, N$. Both tests allow the inclusion of lags of $\Delta y_{i t}$ into the null hypothesis, which makes the test robust for serially correlated errors. Also, the test in Im, Pesaran, and Shin (2003) has a "demeaned" version that is robust when the disturbances are correlated cross-sectionally. In that case the model structure becomes $\Delta \tilde{y}_{i t}=\tilde{\alpha}_{i}+\beta_{i} \tilde{y}_{i, t-1}+\tilde{\varepsilon}_{i t}$, where the tilde above the variables indicates 
that the cross-sectional mean was subtracted from each variable.

Since the tests discussed above require the panel structure to be balanced, we deleted all states with missing observations. The way to construct those tests is explained with detail in the cited articles. All the tests explained above are distributed standard normal under the null hypothesis. Those are lower tail tests, thus the null hypothesis is rejected at $95 \%$ of confidence if the value of the test is less than -1.645 . The panel unit root test results (available on request) show that using the different test specifications, we consistently reject the null hypothesis that the series of the econometric model are $I(1)$ for all the cross-sections of the panel because with very few exceptions, all the $t$-statistics are less than the critical value of -1.645 . Thus, our panel unit root tests do not suggest differencing the data before the estimation.

\section{Panel Data Estimation}

We use the Fixed Effects model for our panel data for two reasons. The primary reason is that the Fixed Effects model allows us to estimate a unit-specific effect for each state in the model. In addition, the Fixed Effects model does not require the restrictive assumption that the state-specific effect is independent of the included covariates as the Random Effects model does. State dummies are included in our regression to capture state-specific effects that are invariant over time. This procedure was applied in all the stages explained in the previous section: in the first-stage OLS estimation, variance estimation, and second-stage WLS estimation.

In addition to the variables we described in the data section, we also included the interaction between temperature and region, reasoning that the effect of higher temperatures is not uniform across regions. Similar interaction terms between precipitation and regional dummies were also included in alternative specifications. Since there appears to be little variation in the effects of precipitation across regions, we decided not to include them in the reported results. Our results, however, are not sensitive to this alternative specification.

The final estimates of the parameters of the proposed stochastic production function is presented in table 1 , where the models are estimated by the FGLS method and the standard errors have been adjusted appropriately to account for the first-stage variation. The functional form for the average yield equation is linear for both the independent and the dependent variables; meanwhile the variance equation is linear for the independent variables but the dependent variable appears logarithmically to assure positive predicted variances. To save space, the coefficients for the individual state dummies are not reported herein. The average yield estimations show that climate affects average yields for cotton and winter wheat through a significant coefficient on precipitation, being positive for cotton and negative for winter wheat. This suggests that holding acreage and all other involved variables constant, a higher amount of total annual precipitation increases cotton yields, decreases winter wheat yields, and does not affect the other crops. Precipitation effects are also covered through the PDSI and intensity independent variables. The PDSI is positive and significant for all crops except for average cotton yields, which respond favorably to lessened drought incidence. The parameter for precipitation intensity is significant and negative for all the crops, suggesting that a shift toward greater intensity-in terms of periods with high amounts of rain while the rest of the year is relatively dry-is harmful for the crops. This result, combined with what we get from precipitation alone, suggests that precipitation intensity and droughts are of greater concern than the annual amount of precipitation alone.

For the independent variables related to temperature, a higher variability in temperature implies a decrease in the yields for all crops, which is consistent with the idea of the negative effect of more extreme eventshigher maximums and lower minimumson agriculture. The variable "Temperature" should be understood as the effect of temperature for the base region (Central), while the coefficients for all of the interaction terms reflect the differences between the temperature effects over a given region with respect to the Central region. Positive (negative) signs indicate a beneficial (harmful) effect of higher temperatures on crop yields. Notice that because some crops are not grown in some regions, some of the regional dummy interaction terms do not appear in the cotton, sorghum, and soybeans equations. It is suggested that temperature has no significant effect over Central regions (positive for soybeans), with negative relative effects for the Southeast and Northeast $(\mathrm{NE})$ regions (for $\mathrm{NE}$ the relative effect 


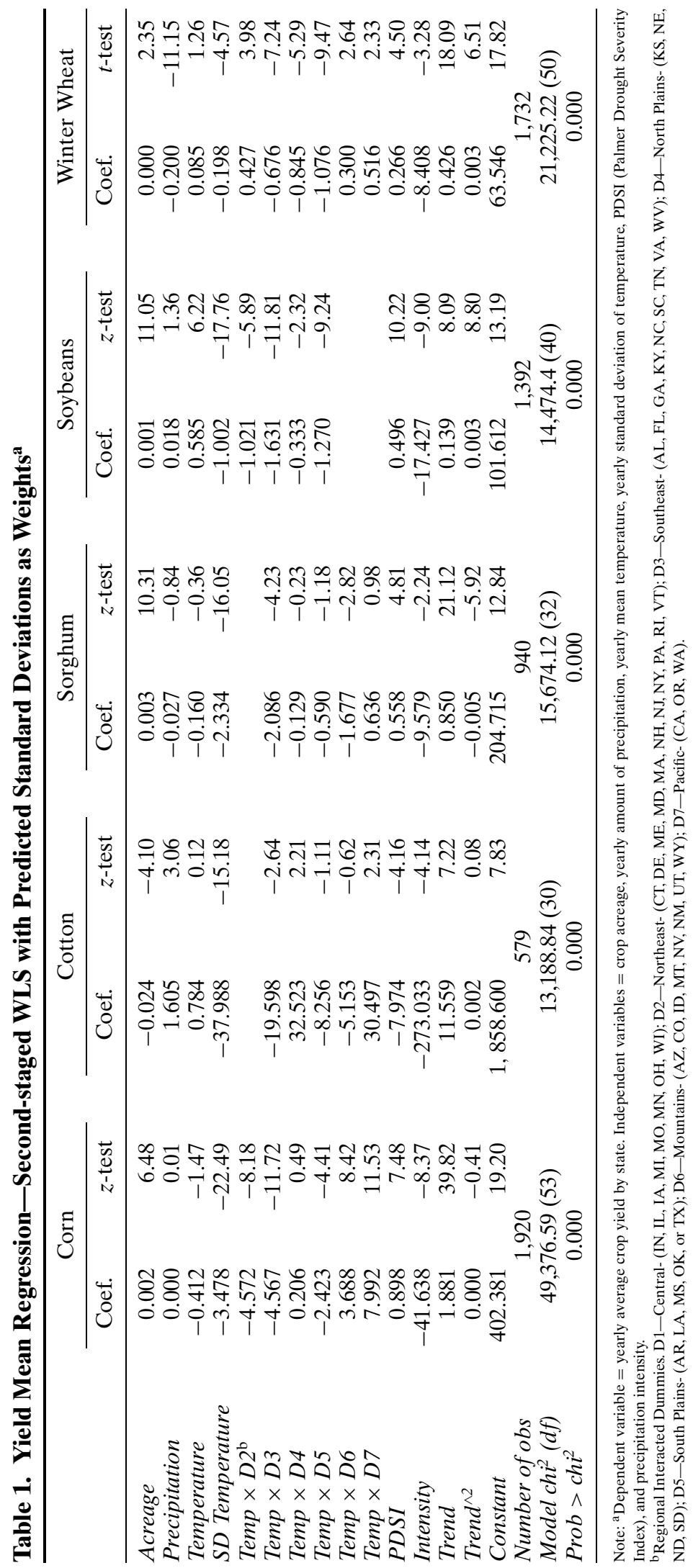




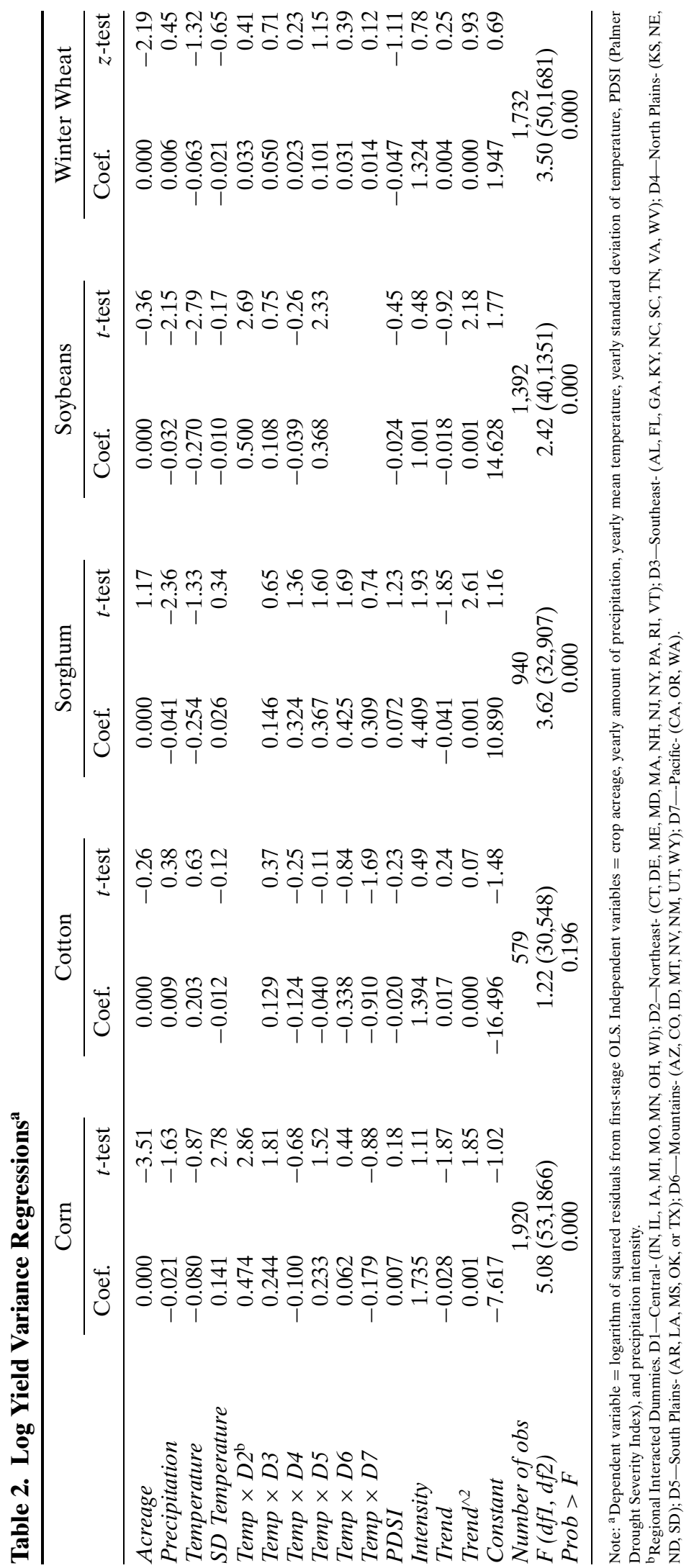


Table 3. Results on Percentage Change in Yield and Yield Standard Deviation under Projections of Climate Change

\begin{tabular}{|c|c|c|c|c|c|c|c|c|c|c|}
\hline \multirow[b]{2}{*}{ Region } & \multicolumn{5}{|c|}{ Canadian } & \multicolumn{5}{|c|}{ Hadley } \\
\hline & Corn & Cotton & Sorghum & Soybeans & $\begin{array}{l}\text { Winter } \\
\text { Wheat }\end{array}$ & Corn & Cotton & Sorghum & Soybeans & $\begin{array}{l}\text { Winter } \\
\text { Wheat }\end{array}$ \\
\hline \multicolumn{11}{|c|}{ Mean yield change with SD temp as GCM predicts } \\
\hline Central $^{\mathrm{a}}$ & 21 & 26 & 2 & 24 & 31 & 21 & 28 & 1 & 26 & 27 \\
\hline Northeast & 32 & & & 18 & 23 & 29 & & & 28 & 25 \\
\hline Southeast & 31 & 21 & -4 & 21 & 28 & 36 & 34 & 2 & 33 & 19 \\
\hline N. Plains & 29 & 57 & 10 & 23 & 38 & 26 & 50 & 6 & 21 & 34 \\
\hline S. Plains & 10 & -4 & -18 & 10 & 46 & 12 & 8 & -15 & 26 & 41 \\
\hline Mountain & 18 & 10 & -4 & & 41 & 12 & 4 & -17 & & 45 \\
\hline Pacific & 15 & 7 & -5 & & 19 & 14 & 4 & -7 & & 19 \\
\hline \multicolumn{11}{|c|}{ Mean yield change with SD temp increased $10 \%$} \\
\hline Central & 22 & 28 & 3 & 25 & 29 & 22 & 30 & 3 & 27 & 26 \\
\hline Northeast & 32 & & & 18 & 23 & 30 & & & 28 & 26 \\
\hline Southeast & 40 & 34 & 9 & 31 & 28 & 39 & 39 & 7 & 36 & 20 \\
\hline N. Plains & 24 & 43 & 4 & 18 & 36 & 24 & 45 & 4 & 20 & 33 \\
\hline S. Plains & 26 & 33 & 6 & 28 & 45 & 24 & 38 & 4 & 40 & 40 \\
\hline Mountain & 23 & 17 & 8 & & 40 & 22 & 17 & 7 & & 42 \\
\hline Pacific & 23 & 18 & 5 & & 19 & 24 & 18 & 6 & & 19 \\
\hline \multicolumn{11}{|c|}{ Standard deviation change with SD temp as GCM predicts } \\
\hline Central & 56 & 65 & 131 & 77 & 68 & 37 & 95 & 67 & 32 & 74 \\
\hline Northeast & 87 & & & 373 & 169 & 56 & & & 121 & 90 \\
\hline Southeast & 99 & -9 & 302 & 234 & 123 & 34 & 19 & 108 & 93 & 120 \\
\hline N. Plains & 61 & -1 & 326 & 257 & 86 & 58 & 12 & 233 & 178 & 87 \\
\hline S. Plains & 173 & 30 & 253 & 140 & 125 & 56 & 202 & -2 & -31 & 81 \\
\hline Mountain & 113 & 7 & 317 & & 115 & 108 & 70 & 139 & & 68 \\
\hline Pacific & 150 & -33 & 503 & & 162 & 223 & -57 & 982 & & 182 \\
\hline \multicolumn{11}{|c|}{ Standard deviation change with SD temp increased $10 \%$} \\
\hline Central & 44 & 66 & 127 & 78 & 58 & 32 & 95 & 66 & 32 & 70 \\
\hline Northeast & 79 & & & 374 & 165 & 53 & & & 121 & 94 \\
\hline Southeast & 60 & -8 & 287 & 240 & 125 & 25 & 20 & 106 & 93 & 129 \\
\hline N. Plains & 76 & -1 & 333 & 255 & 77 & 65 & 12 & 235 & 177 & 80 \\
\hline S. Plains & 62 & 36 & 221 & 149 & 121 & 6 & 212 & -9 & -29 & 77 \\
\hline Mountain & 74 & 9 & 301 & & 112 & 51 & 75 & 125 & & 58 \\
\hline Pacific & 80 & -31 & 468 & & 161 & 124 & -56 & 911 & & 176 \\
\hline
\end{tabular}

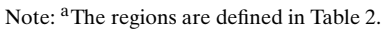

is positive for winter wheat). We get mixed results for the North Plains and negative relative effects for the South Plains (though the relationship is not significant for cotton and sorghum). This indicates the not unexpected results that temperature increases in the hotter areas (the South) are mainly detrimental while increases in the colder (northern) areas are mainly beneficial with the Central areas largely unchanged. Finally, the linear trend is positive and significant for all crops, while the quadratic term is negative for sorghum but positive for soybeans and wheat.

We report the regression results of variance of the residuals from the first stage in table 2. Regarding the variance equation, the interpretation of a positive coefficient implies that an increase in the associated variable leads to a higher yield variance. Notice that for cotton, the joint significance test implies a null effect of all the variables of that model, so cotton yields are found to have a stationary variance. Precipitation negatively affects the log variance of corn, sorghum, and soybeans. Higher temperature decreases log variance for soybeans in Central region, while it increases the relative volatility of corn and soybeans in the Northeast. For South Plains, higher temperatures increase relative log variance of soybean yields. Finally, higher precipitation intensity seems to increase the log variance of sorghum yields.

\section{Simulation of Climate Change Impacts}

In this section, we use simulation methods to evaluate the likely impacts of future climate changes. Parameters estimated from the above 
models are used in this simulation. We first investigate the potential impact of change in average intraannual temperature and precipitation on future crop yield average and variability using projected climate changes from the Hadley and Canadian General Circulation Models (GCM). Next we investigate the combined effects of the above climate changes plus a $10 \%$ increase in temperature variability. The results for year 2030 are summarized in table 3 for all crops and regions. Substantial changes in crop yields and variability arise. The impacts vary across crops and regions. For example, for corn in the central region-the Midwest-under the Canadian model, the projected changes in average climate conditions will increase average corn yield and variation by $21 \%$ and $56 \%$ respectively, compared to $22 \%$ and $44 \%$ if a $10 \%$ increase in temperature variability also occurs. The pattern is somewhat different for corn in the North Plains, where the corresponding changes are $29 \%$ and $61 \%$ for changes in average climate conditions, and $24 \%$ and $76 \%$ when higher temperature variability occurs.

\section{Conclusions}

In this study we investigate the impact of historical climate changes on the stationarity of the crop yield distribution, considering temperature, precipitation, variance of intraannual temperature, a constructed index of rainfall intensity, and PDSI. Our regression results show that stationarity does not hold as we find that both the mean and the variance of key climate variables evolved over time. In turn the average climate conditions and their variability appear to contribute in a statistically significant way to not only average crop yields but to their variability as well. In particular we find that the mean of the crop yields are affected by the average temperature and precipitation. In addition, we also note that higher variances in climate conditions tend to lower average crop yield and inflate yield variability, although the magnitude of this effect varies across crops. The variability of precipitation, as measured by a rainfall intensity index and PDSI, is shown to have a significant impact on crop yields as well.

These results suggest that stationarity is in fact a questionable assumption and that risk analysts should consider this when developing probabilistic models where climate plays an important direct or indirect role. It appears likely that climate change will increase the variability of crop yield distributions, and this means that historical distributions are going to need dynamic updating particularly since the pace of climate change is increasing as indicated by the recent IPCC reports. Stationarity is certainly dying and risk increasing, creating a demand for improved analysis under climaterelated risk.

\section{References}

Adams, R.M., C. Rosenzweig, R.M. Peart, J.T. Ritchie, B.A. McCarl, J.D. Glyer, R.B. Curry, J.W. Jones, K.J. Boote, and L.H. Allen, Jr. 1990. "Global Climate Change and US Agriculture." Nature 345:219-24.

Chen, C., B.A. McCarl, and D.E. Schimmelpfennig. 2004. "Yield Variability as Influenced by Climate: A Statistical Investigation." Climatic Change 66:239-61.

Deschenes, O., and M. Greenstone. 2007. "The Economic Impacts of Climate Change: Evidence from Agricultural Output and Random Fluctuations in Weather." American Economic Review 97(1):354-85.

Granger, C.W.J., and P. Newbold. 1974. "Spurious Regressions in Economics." Journal of Econometrics 2:111-20.

Im, K.S., M.H. Pesaran, and Y. Shin. 2003. "Testing for Unit Roots in Heterogeneous Panels." Journal of Econometrics 115:53-4.

Just, R., and R.D. Pope. 1978. "Stochastic Specification of Production Function and Economic Implications." Journal of Econometrics 7:67-6.

Levin, A., C. Lin, and C. Chu. 2002. "Unit Root Tests in Panel Data: Asymptotic and Finite Sample Properties." Journal of Econometrics 108:1-24.

Mendelsohn, R., W. Nordhaus, and D. Shaw. 1994. "The Impact of Climate on Agriculture: A Richardian Approach." American Economic Review 84:753-71.

Mearns, L.O., C. Rosenzweig, and R. Goldberg. 1992. "Effect of Changes in Interannual Climatic Variability on CERES-Wheat Yields: Sensitive and $2 \times \mathrm{CO}_{2}$ General Circulation Model Studies." Agricultural and Forest Meteorology 62:159-89.

Milly, P.C.D., J. Betancourt, M. Falkenmark, R.M. Hirsch, Z.W. Kudzewicz, D.P. Lettenmaier, and R.J. Stouffer. 2008. "Climate Change: Stationarity is Dead: Whither Water Management?" Science 319:573-74.

Reilly, J.M., J. Graham, D.G. Abler, R. Darwin, S. Hollinger, C. Izaurralde, S. Jagtap, J. Jones, J. Kimble, B. McCarl, L. Mearns, D. Ojima, E.A. Paul, K. Paustian, S. Riha, N. Rosemberg, C. Rosenzweig, and F. Tubiello. 2002. Changing Climate and Changing Agriculture: Report of the Agricultural Sector Assessment Team. Cambridge: Cambridge University Press. 